\title{
The Role of Prestroke Glycemic Control on Severity and Outcome of Acute Ischemic Stroke
}

\author{
Clara Hjalmarsson, ${ }^{1}$ Karin Manhem, ${ }^{2,3}$ Lena Bokemark, ${ }^{2}$ and Björn Andersson ${ }^{2}$ \\ ${ }^{1}$ The Department of Cardiology, Sahlgrenska University Hospital, Blå Stråket 3, 1st Floor, 40530 Göteborg, Sweden \\ ${ }^{2}$ The Stroke Unit, Department of Internal Medicine, Sahlgrenska University Hospital, Blå Stråket 5, 1st Floor, 40530 Göteborg, Sweden \\ ${ }^{3}$ The University of Gothenburg, 40530 Göteborg, Sweden \\ Correspondence should be addressed to Clara Hjalmarsson; clara.hjalmarsson@vgregion.se
}

Received 18 May 2014; Revised 7 August 2014; Accepted 8 August 2014; Published 8 September 2014

Academic Editor: Wai-Kwong Tang

Copyright (C) 2014 Clara Hjalmarsson et al. This is an open access article distributed under the Creative Commons Attribution License, which permits unrestricted use, distribution, and reproduction in any medium, provided the original work is properly cited.

\begin{abstract}
Background/Aim. Relatively few studies have investigated the association of prestroke glycemic control and clinical outcome in acute ischemic stroke (IS) patients, regardless of presence of diabetes mellitus (DM). The aim of this study was to investigate the importance of prestroke glycemic control on survival, stroke severity, and functional outcome of patients with acute IS. Methods. We performed a retrospective survival analysis of 501 patients with IS admitted to Sahlgrenska University Hospital from February 15, 2005, through May 31, 2009. The outcomes of interest were acute and long-term survival; the stroke severity (NIHSS) and the functional outcome, mRS, at 12 months. Results. HbAlc was a good predictor of acute (HR 1.45; CI, 1.09 to $1.93, P=0.011$ ) and long-term mortality (HR 1.29; CI 1.03 to $1.62 ; P=0.029$ ). Furthermore, HbAlc $>6 \%$ was significantly correlated with acute stroke severity (OR 1.29; CI 1.01 to $1.67 ; P=0.042$ ) and predicted worse functional outcome at 12 months (OR 2.68; CI 1.14 to 6.03; $P=0.024)$. Conclusions. Our study suggests that poor glycemic control (baseline HbAlc) prior to IS is an independent risk factor for poor survival and a marker for increased stroke severity and unfavorable long-term functional outcome.
\end{abstract}

\section{Introduction}

Hyperglycemia (HG) in relation to acute IS is common both in patients with and in patients without a diagnosis of DM, and it has been suggested to worsen survival. However, recent results from several clinical studies indicate that particularly patients with stroke and stress HG, but not diabetes, have increased mortality [1-3].

On the contrary, older data by Woo et al. [4] found that patients with acute IS and similar glucose concentrations had similar outcome regardless of whether they had diabetes or not.

According to a review published by Capes et al. [5], acute HG predicted increased risk of in-hospital mortality after ischemic stroke (IS) in nondiabetic patients and increased risk of poor functional recovery in nondiabetic stroke survivors. The recent results of Nardi et al. [2] are also in line with this conclusion.
In a study published in 2012, $\mathrm{Hu}$ et al. [1] evaluated the effects of HG and prestroke glycemic control, as measured by HbAlc, on all-cause and cardiovascular mortality among 1277 IS patients and found a significant association between initial glucose level and mortality in nondiabetic patients. Surprisingly, they also found that DM patients with $\mathrm{HbAlc}<7.0 \%$ had a higher incidence rate of all-cause and cardiovascular death than those with $\mathrm{HbAlc} \geq 7 \%$. Contradictory data have been published by Kamouchi et al. in 2011, showing that patients with acute IS and DM had more unfavorable outcome, the higher HbAlc they had [6].

It is worth pointing out that several of the abovementioned studies compared the patients by the clinical prestroke DM diagnosis, while no evaluation of HbAlc in the whole study cohort was available. As a result, there could have been interference from patients with subclinical DM who got included in the group without a diagnosis of DM. 
A recent study by $\mathrm{Li}$ et al. [7] is one of the few studies systematically investigating the role of HbAlc on stroke outcome, regardless of a prestroke diagnosis of DM; their results show that elevated $\mathrm{HbAlc}$ level relates to stroke severity and poor prognosis in the whole study population; however, only patients with brainstem infarction were included in this study.

The primary aim of this study was to investigate the effect of prestroke glycemic control (glycated hemoglobin, HbAlc) on acute (30 days) and long-term (one year) survival of IS patients who did not undergo thrombolysis. The secondary aim was to investigate the effects of HG and HbAlc on acute stroke severity and on twelve-month functional outcome.

\section{Methods}

2.1. Study Design and Population. Epidemiological and clinical data, as well as laboratory parameters, were prospectively collected from 799 consecutive patients with acute ischemic stroke admitted to Sahlgrenska University Hospital from February 15, 2005, through May 31, 2009. Patients with transient ischemic attack and those with intracerebral hemorrhage were excluded. Also, patients in which HbAlc was not measured on admission $(n=298)$ were excluded. None of the patients included in the study received thrombolytic therapy. The local ethics committee approved the study.

2.2. Data Collection. Epidemiological data were obtained directly from patients, using standardized data collection instruments. When the patient was unable to provide answers, a proxy with knowledge of the subject's history was interviewed.

ECG, CT-scans, and blood tests were carried out on admission. Standard techniques were used to measure blood pressure, glycated hemoglobin, creatinine, fasting blood glucose, and serum lipid levels.

Patients were classified into two groups (DM, nonDM) according to the presence of a prestroke DM diagnosis. Hypertension was defined as systolic blood pressure $>140 \mathrm{~mm} \mathrm{Hg}$ or diastolic blood pressure $>90 \mathrm{~mm} \mathrm{Hg}$ (based on the average of two blood pressure measurements during hospital stay) or a patient's self-report of a history of hypertension or antihypertensive drug use. Diabetes mellitus was defined as the patient's self-report of such a history or use of insulin or a hypoglycaemic drug. The HbAlc data were used either as continuous variable or as dichotomous variable (HbAlc $\leq 6.0 ;>6.0$, according to $[8,9]$ ).

The diagnosis of stroke was assessed according to the World Health Organization definition of stroke [10]. The National Institutes of Health Stroke Scale (NIHSS) was used to assess the severity of the stroke [11].

Based on clinical and neuroimaging findings, stroke subtypes were classified according to the TOAST classification [12].

The modified Rankin Scale (mRS), validated for stroke outcome assessment, was used by a trained physician for assessing the functional outcome at 12 months after stroke. Favourable outcome was defined as mRS scores $0-2$, corresponding to independence with regard to activities of daily living.
2.3. Outcomes. The primary end points of the study were the survival during the acute phase (up to 30 days after admission) and the long-term survival (up to 12 months after stroke debut).

Other outcomes of interest (secondary end points) were the initial stroke severity defined according to the NIHSS, used as continuous or dichotomized (NIHSS $<7$ and $\geq 7$ ) variable, as appropriate, and the functional outcome, mRS, at 12 months, dichotomized as $\mathrm{mRS} \leq 2$ and $>2$.

2.4. Statistical Analysis. We used the Mann-Whitney $U$ test for comparing the mean values of continuous variables (NIHSS, serum lipid levels, blood glucose, and blood pressure) by the DM group, while the chi-square test was used for discrete variables.

The associations between glycated hemoglobin on admission and stroke severity (number of points according to NIHSS, dichotomous NIHSS $<7$ and $\geq 7$ ), as well as functional outcome at 12 months (dichotomous, $\mathrm{mRS} \leq 2$ and $>2$ ), were investigated using a logistic regression model, adjusting for age, gender, stroke subtype (TOAST criteria), admission blood glucose, previous TIA/stroke, and associated cardiovascular pathology. The same regression model was used for testing the relation between the above-mentioned outcome variables (NIHSS; mRS) and fasting blood glucose (continuous variable) on admission to hospital.

Survival was investigated using the Cox proportional hazard regression, where age, gender, stroke severity (as a continuous variable), stroke subtype, blood glucose, and prestroke medical conditions (previous TIA/stroke, previous myocardial infarction, atrial fibrillation, and DM) were used as covariates.

The log rank test (Mantel-Cox) was used to test the difference in equality distribution for the Kaplan-Meier curves. The results are presented as the hazard or odds ratio (HR, OR) with $95 \%$ confidence intervals (CI). $P$ values $<0.05$ were regarded as statistically significant (2-sided test). All statistical analyses were performed using the SPSS 17.0 package (SPSS Inc., Chicago, IL, USA).

\section{Results}

3.1. Baseline Characteristics. The final dataset consisted of 501 patients. The mean age of the patients included in the analysis was 78.8 years and $48.5 \%$ were men. Table 1 summarizes the demographics and baseline clinical characteristics of our study population in relation to prestroke diagnosed DM; we found 97 patients with DM; of these, 72 patients had type 2 DM; 68 patients were treated with per oral antidiabetics and 31 with insulin. No difference in stroke etiology was found between the DM versus the non-DM group.

There was no difference in mean systolic and diastolic blood pressure on admission between the two groups. However, the patients with DM were significantly younger $(P=$ $0.013)$ and more often had prestroke hyperlipidemia $(P<$ $0.001)$ and prestroke myocardial infarction $(P=0.024)$. The DM patients had significantly higher glycemia, HbAlc, total s-cholesterol, and s-triglycerides on admission; for details see Table 1 . 
TABLE 1: Patients' characteristics at baseline $(N=501)$ by presence of diabetes mellitus.

\begin{tabular}{|c|c|c|c|}
\hline Baseline characteristics & $\begin{array}{l}\text { No diabetes mellitus }(n=404) \\
\qquad N(\%)\end{array}$ & $\begin{array}{l}\text { Diabetes mellitus }(n=97) \\
\qquad N(\%)\end{array}$ & $P$ value \\
\hline Gender, female & $219(54.2)$ & $39(40.2)$ & $0.013^{*}$ \\
\hline Age, years (SD) & $79.2(7.8)$ & $76.5(8.8)$ & $0.003^{* * a}$ \\
\hline Hypertension & $231(57.2)$ & $64(66.0)$ & 0.114 \\
\hline Hyperlipidemia & $52(12.9)$ & $26(26.8)$ & $0.001^{* * *}$ \\
\hline Atrial fibrillation & $139(34.4)$ & $29(29.9)$ & 0.398 \\
\hline Heart failure & $48(11.9)$ & $15(15.5)$ & 0.344 \\
\hline Previous myocardial infarction & $51(12.7)$ & $21(21.6)$ & $0.024^{*}$ \\
\hline Previous intracerebral hemorrhage & $7(1.7)$ & $1(1.0)$ & 0.617 \\
\hline Previous ischemic stroke & $99(24.5)$ & $32(33.0)$ & 0.088 \\
\hline Previous TIA & $26(6.4)$ & $10(10.3)$ & 0.187 \\
\hline \multicolumn{4}{|l|}{ Stroke subtype (TOAST) } \\
\hline Atherothrombotic & $92(22.7)$ & $31(31.9)$ & 0.053 \\
\hline Cardioembolic & $151(37.4)$ & $28(28.9)$ & 0.127 \\
\hline Small vessel disease & $109(27.0)$ & $23(23.7)$ & 0.537 \\
\hline Other causes & $3(0.7)$ & 0 & 0.396 \\
\hline Unknown cause & 48 (11.9) & $14(14.4)$ & 0.476 \\
\hline \multicolumn{4}{|l|}{ Assessments, mean (SD) } \\
\hline Plasma glucose, mean (SD), mmol/L & $7.1(1.8)$ & $10.2(3.9)$ & $<0.001^{* * * a}$ \\
\hline HbAlc (SD), \% & $4.9(0.7)$ & $6.5(1.6)$ & $<0.001^{* * * a}$ \\
\hline Systolic blood pressure, mean (SD), mmHg & $166.8(31.1)$ & $160.6(25.7)$ & $0.079^{\mathrm{a}}$ \\
\hline Diastolic blood pressure, mean (SD), mmHg & $92.7(16.5)$ & $86.0(17.2)$ & $0.001^{* * * a}$ \\
\hline S-cholesterol, total (SD), mmol/L & $4.3(1.1)$ & $5.0(1.2)$ & $<0.001^{* * * a}$ \\
\hline S-LDL (SD), mmol/L & $3.1(1.0)$ & $2.8(2.5)$ & $0.097^{\mathrm{a}}$ \\
\hline S-HDL (SD), mmol/L & $1.5(0.5)$ & $1.3(0.5)$ & $<0.001^{* * * a}$ \\
\hline $\mathrm{S}-\mathrm{TG}(\mathrm{SD}), \mathrm{mmol} / \mathrm{L}$ & $1.2(0.6)$ & $1.5(0.8)$ & $<0.001^{* * * a}$ \\
\hline NIHSS, mean (SD) & $6.5(6.8)$ & $5.8(7.0)$ & $0.391^{\mathrm{a}}$ \\
\hline NIHSS $>7$ & $109(26.9)$ & $19(19.6)$ & 0.094 \\
\hline
\end{tabular}

$P$ values, according to chi-square test.

${ }^{a}$ Mann-Whitney $U$ test.

${ }^{*} P<0.05 ;{ }^{* *} P<0.01 ;{ }^{* * *} P<0.001$.

3.2. Hyperglycemia. Three hundred ninety patients had glycemia above $7 \mathrm{mmol} / \mathrm{L}$ on admission; of these, 87 patients had known prestroke DM. The mean blood glucose level on admission to hospital was significantly higher $(P<0.001)$ in the patients with DM compared with the non-DM patients; for details see Table 1 .

3.3. Glycated Hemoglobin (HbAlc). In the entire study population, $13.2 \%$ had $\mathrm{HbAlc}>6 \%$ ( $\min 3.1 \%$, $\max 14.9 \%$ ) on admission to hospital. About $50 \%$ of the patients with diabetes had $\mathrm{HbAlc}>6.0 \%$, while a total of seventeen patients with $\mathrm{HbAlc}>6.0 \%$ were identified among those without a previously known diagnosis of DM.

\subsection{Primary End Points: Acute and Long-Term Survival}

3.4.1. Acute Survival. The 30-day all-cause mortality rate was $7.8 \%(n=39)$. In a survival analysis by Cox proportional hazard model, the level of HbAlc, used as continuous variable, was a strong predictor of acute mortality (HR 1.45; CI, 1.09 to $1.93, P=0.011$ ), after adjusting for relevant covariates: age, stroke severity, stroke subtype, atrial fibrillation, previously diagnosed DM, blood glucose, and previous TIA/stroke. Moreover, HbAlc $>6 \%$ was also a robust predictor of increased mortality (HR 6.72; CI, 2.02 to 22.34, $P=0.002$ ); for details see Figure 1 and Table 2 .

Glycemia on admission influenced the acute survival in a significant manner (HR 1.16; CI, 1.02 to 1.32; $P=0.028$ ), independent of age, stroke severity, previous TIA/stroke, previously diagnosed DM, and atrial fibrillation. However, when adjusting for prestroke glycemic control (HbAlc), hyperglycemia was no longer an independent predictor.

3.4.2.12-Month Survival. Ninety-eight deaths were registered during the first year after stroke (cumulative mortality rate 19.6\%). The level of HbAlc (HR 1.29; CI 1.03 to 1.62; $P=$ 0.029 ) on admission, used as continuous variable, was an independent predictor of 12-month survival after adjusting 
TABLE 2: Multiple logistic regression analysis showing the relationship between survival and explanatory variables.

\begin{tabular}{|c|c|c|c|c|c|c|}
\hline \multirow{2}{*}{ Explanatory variable } & \multicolumn{3}{|c|}{30 days after stroke } & \multicolumn{3}{|c|}{ One year after stroke } \\
\hline & $\mathrm{HR}^{*}$ & $95 \% \mathrm{CI}$ & $P$ value & $\mathrm{HR}^{*}$ & $95 \% \mathrm{CI}$ & $P$ value \\
\hline Age (years) & 1.04 & $0.96-1.12$ & 0.321 & 1.05 & $1.01-1.09$ & 0.037 \\
\hline Gender, female & 0.70 & $0.31-1.62$ & 0.407 & 0.71 & $0.40-1.24$ & 0.223 \\
\hline Diabetes mellitus & 6.15 & $1.15-32.88$ & 0.034 & 3.06 & $1.07-8.73$ & 0.037 \\
\hline Fasting blood glucose & 1.12 & $0.89-1.41$ & 0.344 & 1.08 & $0.94-1.25$ & 0.273 \\
\hline HbAlc & 6.72 & $2.02-22.34$ & 0.002 & 3.40 & $1.40-8.22$ & 0.007 \\
\hline Atrial fibrillation & 1.20 & $0.48-3.02$ & 0.698 & 1.21 & $0.68-2.16$ & 0.525 \\
\hline Previous myocardial infarction & 0.39 & $0.09-1.69$ & 0.206 & 1.16 & $0.57-2.34$ & 0.681 \\
\hline Previous TIA/ischemic stroke & 2.24 & $0.95-5.31$ & 0.067 & 1.55 & $0.87-2.75$ & 0.134 \\
\hline Stroke severity (NIHSS) & 1.15 & $1.10-1.20$ & $<0.001$ & 1.10 & $1.07-1.13$ & $<0.001$ \\
\hline
\end{tabular}

${ }^{*} \mathrm{HR}$ for increase of age by one year or presence of a specific medical condition.

$\mathrm{HR}<1$ indicates factor favours survival; HR $>1$ indicates factor is associated with poor survival.

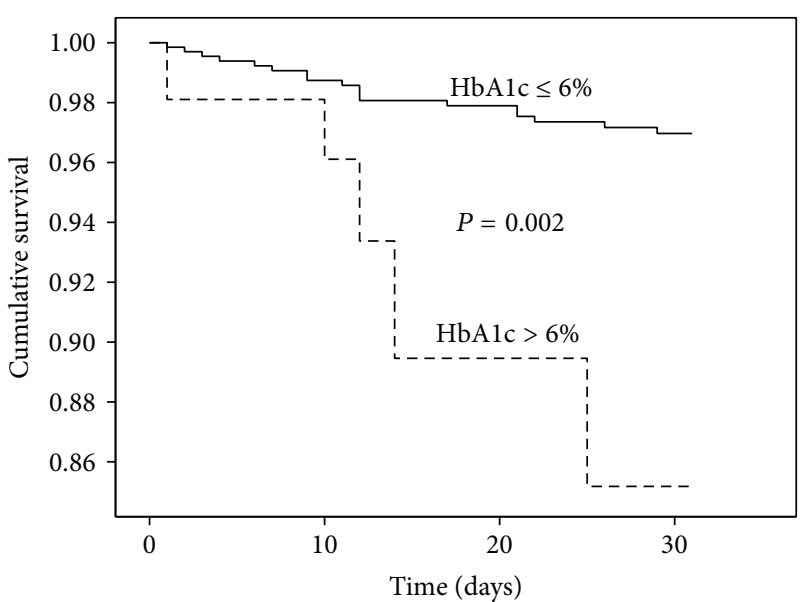

FIGURE 1: Cumulative survival during the first month after acute ischemic stroke ( $n=39$ deaths); stratification according to level of glycated haemoglobin, HbAlc.

for the relevant covariates mentioned above. Also, when dichotomized, HbAlc $>6 \%$ was significantly associated with worse survival (HR 3.40; CI 1.40 to $8.22 ; P=0.007$ ); for details see Figure 2 and Table 2. Glycemia was associated with long-term survival (HR 1.12; CI 1.01 to $1.24 ; P=0.031$ ) but lost its predictive value when the analysis was adjusted for the prestroke glycemic control (HbAlc).

\subsection{Secondary End Points: Stroke Severity and Functional Outcome}

3.5.1. Stroke Severity. In an age-, gender-, stroke-, subtype-, and comorbidity-adjusted linear logistic regression, HbAlc, but not glycemia, was significantly correlated with acute stroke severity (NIHSS $\geq 7$; OR 1.29; CI 1.01 to $1.67 ; P=$ $0.042)$; other significant predictors of stroke severity were age $(P=0.006)$, gender $(P=0.026$, lower risk for men $)$, atrial fibrillation $(P<0.001)$, and prestroke DM $(P=0.044)$.

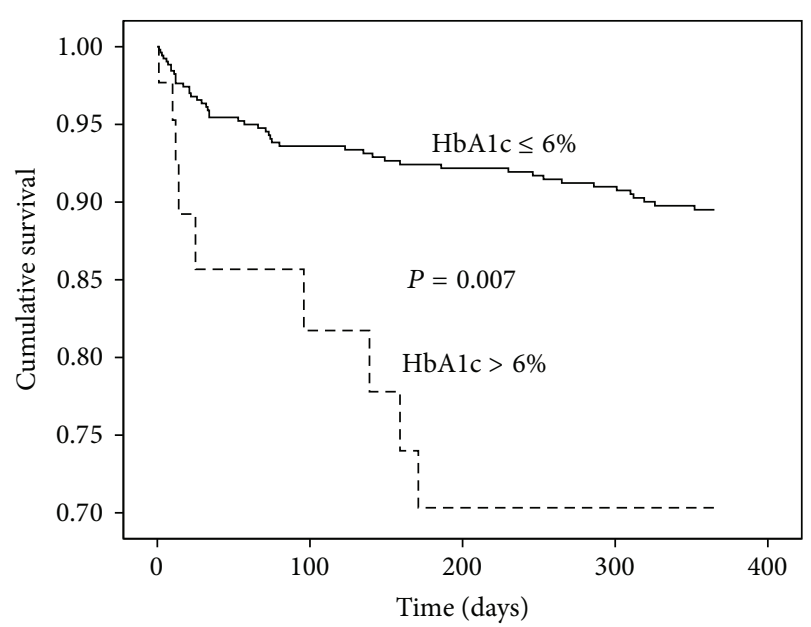

FIGURE 2: Cumulative survival during the 12-month followup after admission to hospital ( $n=98$ deaths); stratification according to level of glycated haemoglobin, HbAlc.

3.5.2. Functional Outcome. The HbAlc $>6 \%$ predicted worse functional outcome ( $\mathrm{mRS}>2$ ) at 12 months after stroke (OR 2.68; CI 1.14 to $6.03 ; P=0.024)$ in a binary regression analysis adjusted for age (OR 1.07; CI 1.04 to $1.12 ; P<0.001$ ), previous stroke/TIA (OR1.99; CI 1.09 to $3.62 ; P=0.025$ ), and stroke severity (OR 1.24; CI 1.17 to $1.31 ; P<0.001$ ); gender, stroke subtype, and glycemia on admission were not related to functional outcome, as defined above.

\section{Discussion}

This is one of the few clinical studies where the role of glycated hemoglobin is systematically evaluated with respect to early and late outcome after IS regardless of whether patients had DM or not. Our results demonstrate that poor prestroke glycemic control is (1) an independent determinant of stroke severity, (2) a good predictor of acute and long-term survival, and (3) a robust marker of neurological functional outcome. 
A large percentage of our patients (77.8\%) had HG on admission, but only $19.4 \%$ had prestroke DM; thus, "stress HG" in the absence of DM is very common.

Several previous studies have found worse survival in patients with $\mathrm{HG}[1,2,4,5,13]$. Some research groups $[1,2,5]$ have reported that $\mathrm{HG}$ is a determinant of worse clinical outcome in patients with IS without DM, but not in those with DM, while others [13] have found a deleterious effect of $\mathrm{HG}$ on survival regardless whether or not the patients had DM. In our study, HG was related to increased mortality, but it lost its predictive value when the analysis was adjusted for prestroke glycemic control. Also, HG was not correlated with stroke severity in an independent manner, even though it has preliminarily been linked to induction of procoagulant state and to neurotoxicity, due to prooxidative and proinflammatory effects [14]. HG did not influence the functional outcome one year after stroke either.

In this work, we found a strong relationship between prestroke glycemic control and neurological outcome, as well as survival. HbAlc was a good independent predictor of stroke severity in the whole study group, not only in patients with DM. Moreover, we observed a fine incremental effect, testing the HbAlc as a continuous variable as well, not only as a categorical one.

Results from two newly published studies $[15,16]$ also show that high HbAlc is independently associated with poor outcome 1 year after stroke, supporting our findings. Nevertheless, one of the studies, based on data from the Fukuoka Stroke Registry [15], only included patients with known DM; moreover, none of the studies investigated the effect of HbAlc on acute stroke severity.

The exact mechanism by which poor prestroke glycemic control affects survival of stroke patients is less clear; general complications related to poorly controlled DM could be one explanation. An increased HbAlc level reflects poor longterm glycemic control and has specific implications for the structure and function of the vascular bed, including small and large cerebral vessels. Increased $\mathrm{HbAlc}$ level might also be a marker of poor compliance, indicating an unhealthy lifestyle.

In our study, no relationship between HbAlc and the type of cerebrovascular lesion, as defined by TOAST criteria, was observed (data not shown). This finding is in line with the results of Heo et al., [17] who did not find any differences in the incidence of large artery disease, leukoaraiosis, cerebral microbleeds, and/or old lacunes by the level of HbAlc in patients with acute ischemic stroke. Thus, even though one would expect patients with diabetes to be more prone to developing stroke secondary to large artery disease and/or small vessel disease, no differences were noted in this study group. Of course, an explanation could be that our study population is quite old and, therefore, has a pronounced cardiac comorbidity, which may even out the potential differences.

One of the limitations of this study is that we did not measure the size of the stroke lesion by CT or MRI; however, it is well known that the NIHSS score is a good clinical outcome measure, which parallels infarct volume [18].
Another limitation is related to the lack of data on the duration of diabetes and serial measurements of the HbAlc during the follow-up period.

In summary, our study demonstrates that impaired prestroke glycemic control (baseline HbAlc) is an independent risk factor for poor survival and for unfavorable functional outcome after IS. Some relatively recent randomized controlled trials [19-21] have found that intensive glycemic control could not reduce cardiovascular risk in diabetic patients, but it is important to highlight that these studies used a cutoff HbAlc higher than 6.4\%. Therefore, additional studies are needed to elucidate whether intensified prestroke glycemic control may improve clinical course and outcome in patients with acute ischemic stroke.

\section{Conflict of Interests}

The authors declare that there is no conflict of interests regarding the publication of this paper.

\section{Acknowledgments}

The authors are greatly indebted to Alieh Shadman and Jessica Antonsson, research nurses, for help with management of the database. This work was partially supported by Research Funds from the County of Västra Götaland, Sweden.

\section{References}

[1] G. C. Hu, S. F. Hsieh, Y. M. Chen, H. Hsu, Y. Hu, and K. Chien, "Relationship of initial glucose level and all-cause death in patients with ischaemic stroke: the roles of diabetes mellitus and glycated hemoglobin level," European Journal of Neurology, vol. 19, no. 6, pp. 884-891, 2012.

[2] K. Nardi, P. Milia, P. Eusebi, M. Paciaroni, V. Caso, and G. Agnelli, "Predictive value of admission blood glucose level on short-term mortality in acute cerebral ischemia," Journal of Diabetes and Its Complications, vol. 26, no. 2, pp. 70-76, 2012.

[3] T. Shimoyama, K. Kimura, J. Uemura et al., "Elevated glucose level adversely affects infarct volume growth and neurological deterioration in non-diabetic stroke patients, but not diabetic stroke patients," European Journal of Neurology, vol. 21, no. 3, pp. 402-410, 2014.

[4] J. Woo, C. W. K. Lam, R. Kay, A. H. Y. Wong, R. Teoh, and M. G. Nicholls, "The influence of hyperglycemia and diabetes mellitus on immediate and 3-month morbidity and mortality after acute stroke," Archives of Neurology, vol. 47, no. 11, pp. 1174-1177, 1990.

[5] S. E. Capes, D. Hunt, K. Malmberg, P. Pathak, and H. C. Gerstein, "Stress hyperglycemia and prognosis of stroke in nondiabetic and diabetic patients: a systematic overview," Stroke, vol. 32, no. 10, pp. 2426-2432, 2001.

[6] M. Kamouchi, T. Matsuki, J. Hata et al., "Prestroke glycemic control is associated with the functional outcome in acute ischemic stroke: the fukuoka stroke registry," Stroke, vol. 42, no. 10, pp. 2788-2794, 2011.

[7] H. Li, Z. Kang, W. Qiu et al., "Hemoglobin A1C is independently associated with severity and prognosis of brainstem infarctions," Journal of the Neurological Sciences, vol. 317, no. 1-2, pp. 87-91, 2012. 
[8] R. A. Silverman, U. Thakker, T. Ellman et al., "Hemoglobin $A_{1 c}$ as a screen for previously undiagnosed prediabetes and diabetes in an acute-care setting," Diabetes Care, vol. 34, no. 9, pp. 19081912, 2011.

[9] T. Tankova, N. Chakarova, L. Dakovska, and I. Atanassova, "Assessment of HbAlc as a diagnostic tool in diabetes and prediabetes," Acta Diabetologica, vol. 49, no. 5, pp. 371-378, 2012.

[10] "The World Health Organization MONICA Project (monitoring trends and determinants in cardiovascular disease): a major international collaboration. WHO MONICA Project Principal Investigators," Journal of Clinical Epidemiology, vol. 41, no. 2, pp. 105-114, 1988.

[11] T. Brott, H. P. Adams Jr., C. P. Olinger et al., "Measurements of acute cerebral infarction: a clinical examination scale," Stroke, vol. 20, no. 7, pp. 864-870, 1989.

[12] H. P. Adams Jr., B. H. Bendixen, L. J. Kappelle et al., "Classification of subtype of acute ischemic stroke: definitions for use in a multicenter clinical trial," Stroke, vol. 24, no. 1, pp. 35-41, 1993.

[13] L. S. Williams, J. Rotich, R. Qi et al., "Effects of admission hyperglycemia on mortality and costs in acute ischemic stroke," Neurology, vol. 59, no. 1, pp. 67-71, 2002.

[14] R. Garg, A. Chaudhuri, F. Munschauer, and P. Dandona, "Hyperglycemia, insulin, and acute ischemic stroke: a mechanistic justification for a trial of insulin infusion therapy," Stroke, vol. 37, no. 1, pp. 267-273, 2006.

[15] T. Kuwashiro, H. Sugimori, T. Ago, J. Kuroda, M. Kamouchi, and T. Kitazono, "The impact of predisposing factors on longterm outcome after stroke in diabetic patients: the Fukuoka Stroke Registry," European Journal of Neurology, vol. 20, no. 6, pp. 921-927, 2013.

[16] S. Wu, C. Wang, Q. Jia et al., "HbAlc is associated with increased all-cause mortality in the first year after acute ischemic stroke," Neurological Research, vol. 36, pp. 444-452, 2014.

[17] S. H. Heo, S. Lee, B. J. Kim, B. S. Kang, and B. W. Yoon, "Does glycated hemoglobin have clinical significance in ischemic stroke patients?" Clinical Neurology and Neurosurgery, vol. 112, no. 2, pp. 98-102, 2010.

[18] D. C. Tong, M. A. Yenari, G. W. Albers, M. O’Brien, M. P. Marks, and M. E. Moseley, "Correlation of perfusion- and diffusionweighted MRI with NIHSS score in acute ( $<6.5$ hour) ischemic stroke," Neurology, vol. 50, no. 4, pp. 864-870, 1998.

[19] W. Duckworth, C. Abraira, T. Moritz et al., "Glucose control and vascular complications in veterans with type 2 diabetes," The New England Journal of Medicine, vol. 360, no. 2, pp. 129-139, 2009.

[20] H. C. Gerstein, M. E. Miller, R. P. Byington et al., "Effects of intensive glucose lowering in type 2 diabetes," The New England Journal of Medicine, vol. 358, no. 24, pp. 2545-2559, 2008.

[21] A. Patel, S. MacMahon, J. Chalmers et al., "Intensive blood glucose control and vascular outcomes in patients with type 2 diabetes," The New England Journal of Medicine, vol. 358, no. 24, pp. 2560-2572, 2008. 


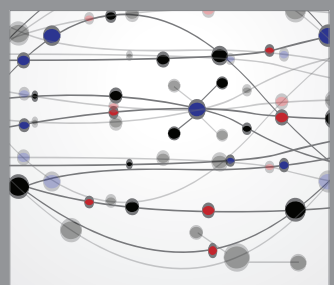

The Scientific World Journal
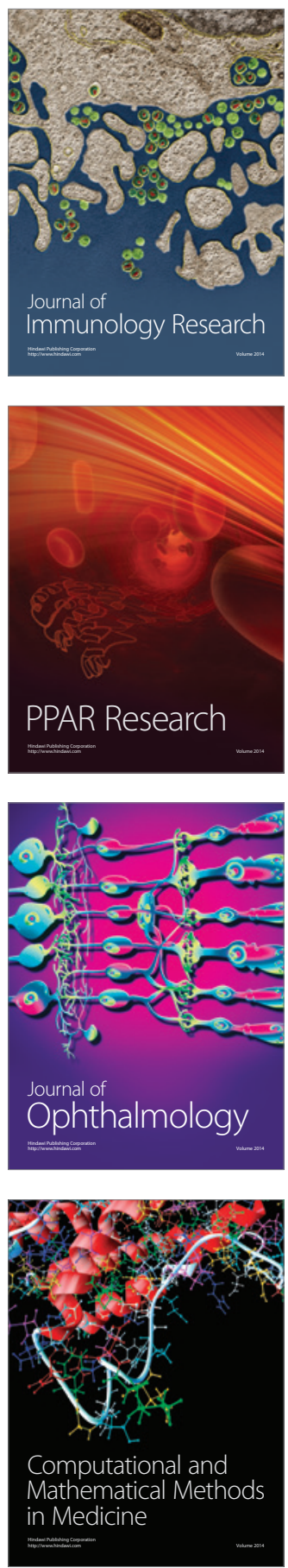

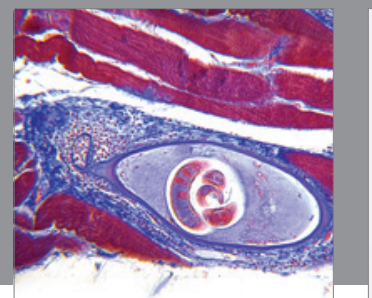

Gastroenterology

Research and Practice
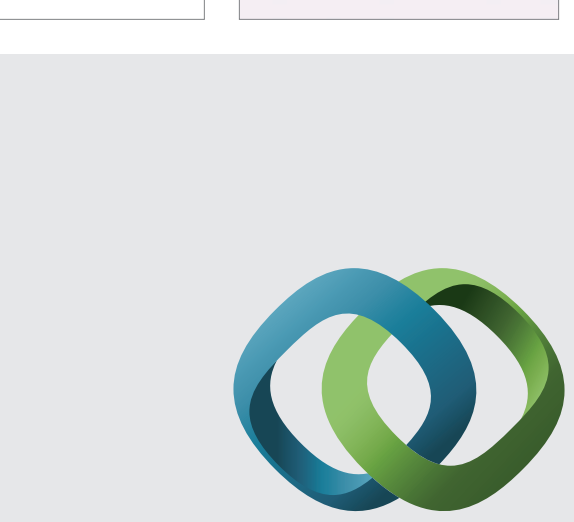

\section{Hindawi}

Submit your manuscripts at

http://www.hindawi.com
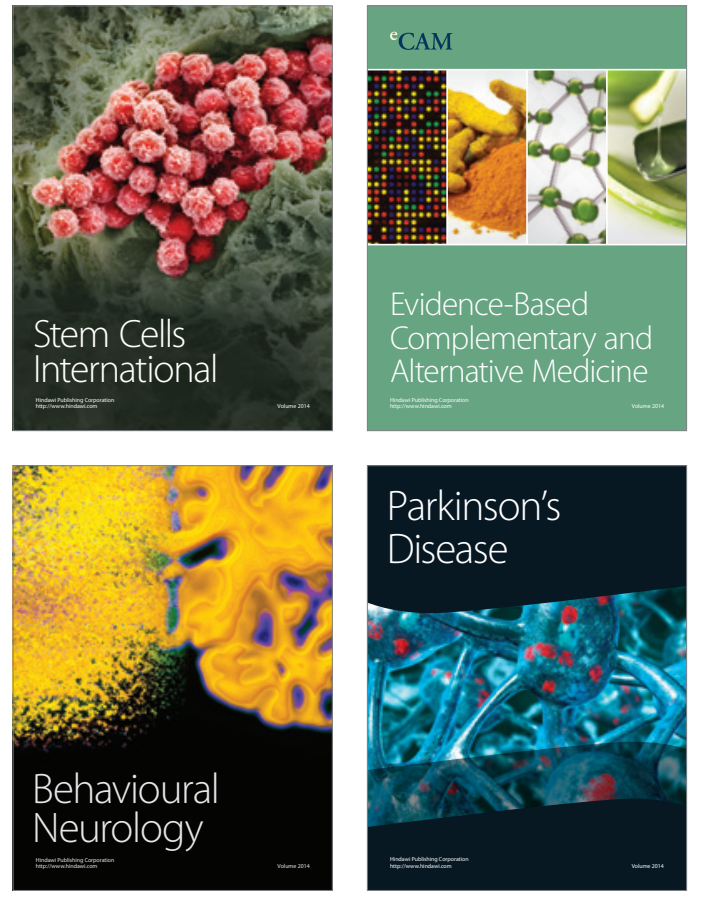
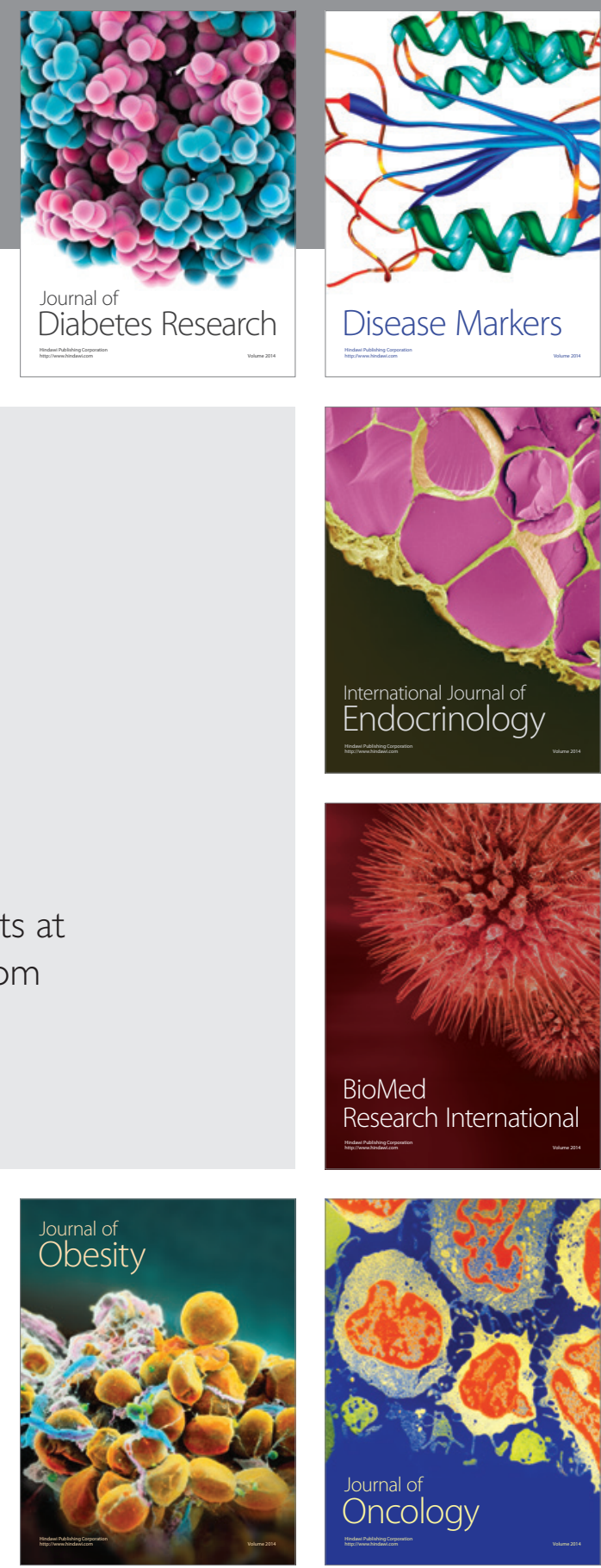

Disease Markers
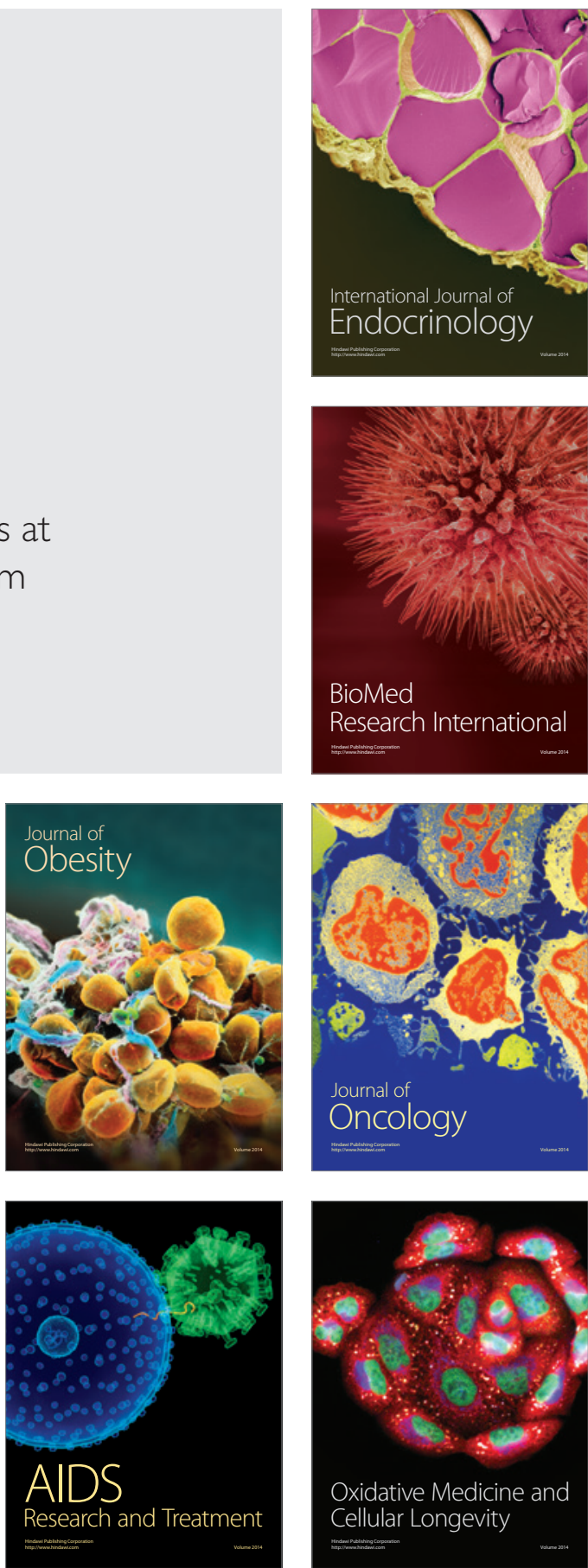